\title{
CRS AND GUARDED LOGICS: A FRUITFUL CONTACT
}

Johan van Benthem, Amsterdam \& Stanford

http://staff.science.uva.nl/ johan

January 2009

\section{Introduction: back and forth between algebra and model theory}

Algebra and model theory are complementary stances in the history of logic, and their interaction continues to spawn new ideas, witness the interface of First-Order Logic and Cylindric Algebra. This chapter is about a more specialized contact: the flow of ideas between algebra and modal logic through 'guarded fragments' restricting the range of quantification over objects. Here is some general background for this topic. For a start, the connection between algebra and model theory is rather tight, since we can view universal algebra as the equational logic part of standard first-order model theory. As an illustration, van Benthem 1988 has a purely model-theoretic proof of Jónsson's Theorem characterizing the equational varieties with distributive lattices of congruence relations, a major tool of algebraists. Deeper connections arise in concrete cases with categorial dualities, such as that between BAOs and the usual relational models of modal logic. An important example is the main theorem in Goldblatt \& Thomason 1974 characterizing the elementary modally definable frame classes through their closure under taking generated sub-frames, disjoint unions, $p$-morphic images, and anti-closure under ultrafilter extensions. Its original proof goes back and forth between algebras and frames, in order to apply Birkhoff's characterization of equational varieties. Later on, van Benthem 1993 gave a purely model-theoretic proof, replacing trips into algebraic territory by the use of $\omega$-saturated models. Even so, the trade between algebra and logic remains interesting, even when it is not a matter of applying concrete theorems, but exporting more general ideas. The present chapter is a case in point. So-called relativization started as a technique for generalizing relational and cylindric algebra, while also, in some cases, 'defusing' the undecidability of these systems. But as we shall see, mainly based on van Benthem 1996A, Andréka, van Benthem \& Németi 1998, van Benthem 2005, it has traveled well into standard logic and model theory. 


\section{From CRS and relational algebra to modal arrow logic}

\subsection{Relativization: technique and motivation}

Relativization Relativization in relational algebra restricts the available pairs on an underlying set $Y$ to some subset, i.e., some largest binary relation $U$, not necessarily the full product $Y \times Y$. Computation of values for complex algebraic terms still proceeds via the usual operations of complementation, union, composition, etc. but with their clauses relativized to work inside the set $U$. In particular, with compositions, we have a clause

$x R ; S y$ iff there exist pairs $(x, z),(z, y) \in U$ such that $x R z$ and $z S y$.

This extension of the set of models leaves several base laws of relational algebra valid, while others become invalid: Associativity $(R S) ; T=R ;(S ; T))$ is a typical example. Crucially, in this contraction process, the set of algebraic validities becomes decidable. But if we impose additional conditions on the relation $U$, then undecidability may reappears. For instance, if we require transitivity, relational algebra is undecidable again: the set of available pairs then looks 'too much' like the full Cartesian product $Y x Y$. For further details, we refer to Németi 1985, 1995 as well as several chapters in this Book. The system CRS, another product of the well-known 'Budapest School' of Hajnal Andréka, Ístvan Németi and their students develops analogous ideas for all of cylindric algebra, with similar effects - and it, too, has generated a large subsequent literature (cf. Venema 1991, Marx 1995, Mikulas 1995, Marx \& Venema 1997, Venema 2006).

These ideas have counterparts in logic, and they have been influential in several ways. Relativization in relational algebra suggests a modal perspective where transitions are now viewed as objects in their own right ('arrows'), in addition to points or states, while algebraic terms now correspond to modal formulas defining properties of transitions. For the development of this 'Arrow Logic', cf. van Benthem 1991, Venema 1991, 1996. Likewise, CRS has influenced logical systems, in particular 'cylindric modal logic' (Venema 1995) and 'first-order dependence logic' (van Benthem 1997B), where gaps in the total space of variable assignments model the important phenomena of dependence and independence between variables, that have come to the fore recently with many authors (van den Berg 1996, Hodges 2001, Väänänen 2007). Both lines from algebra to logic, arrow logic and dependence logics, will be discussed in this chapter. 
Generalized models Extending original classes of models for logics to manipulate their properties is widespread. The famous move from 'standard models' to 'general models' in Henkin 1949 turns the complex system of second-order logic into an axiomatizable two-sorted first-order logic (van Benthem \& Doets 1983). Such moves are most attractive when they get an independent motivation. For relational algebra and cylindric algebra, this is provided by what van Benthem 1996A called 'content versus wrappings' in logical modeling. Intuitively, the core calculus of action embodied in relational algebra seems simple, and undecidability comes as a surprise. Thus, we want to find a semantics that gives just the bare bones of action, while additional effects of "standard set-theoretic modeling' are separated out as negotiable decisions of formulation that engender the undecidability. This theme underlies the systems presented in this chapter.

Fragments But there is also a quite different technical way of viewing relativization as a general logical device. Already Wadge 1975 showed how relational algebra can be axiomatized smoothly by using explicit pair notation $(x, y): R$, making transitions explicit as objects, which suggests viewing it as a fragment of first-order logic. Now it is a well-known result of Tarski's that standard relational algebra translates into the undecidable 3-variable fragment of first-order logic, through clauses such as

$$
R ; S(x, y) \leftrightarrow \exists z(R(x, z) \wedge S(z, y))
$$

which typically use existential quantification over objects in the domain. But the clause in our earlier description replaces this by another syntactic format, namely

$$
R ; S(x, y) \leftrightarrow \exists z(\boldsymbol{U}(\boldsymbol{x}, z) \wedge \boldsymbol{U}(z, y) \wedge R(x, z) \wedge S(z, y))
$$

Thus, we end up inside a sub-language of the 3-variable fragment, where patterns of quantification are restricted or 'guarded' in some way by atomic formulas. Similar points hold for $C R S$ and first-order dependence logics, and the result there is that we end up in a sub-language of full first-order logic known as the Guarded Fragment.

In this paper, we will develop this fragment view as well, and eventually, we will also address the following fundamental question about our presentation so far. What is the relation between the two lines of (a) taking a logical language and extending its class of models, and (b) retaining the original model class while restricting the language? 


\subsection{Arrow logic in a nutshell}

Motivation: core content versus wrappings Relational algebra is a calculus of transition relations modeling actions in general. But then its undecidability raises an issue, since basic action does not seem to involve high complexity. We want a dynamic core logic avoiding spurious complexity of 'wrappings': accidents of formulation. This motivates Arrow Logic (van Benthem 1991, Venema 1991, 1996), inspired by the CRS version of relational algebra, taking transitions seriously as objects in their own right.

Models and language Intuitively, binary relations denote sets of arrows. Think of 'arcs' in multi-graphs, 'transitions' for dynamic procedures in computer science, or 'preferences'. Arrows may have internal structure beyond ordered pairs <source state, target state $>$ : several arrows may share one input-output pair, but also certain pairs may not be instantiated by an arrow. This motivates the following abstract notion:

Definition Arrow Frames.

Arrow Frames $\boldsymbol{F}=\left(A, C^{3}, R^{2}, I^{1}\right)$ have objects $A$ ('arrows') with predicates $C^{3} x, y z(x$ is a 'composition' of $y, z), R^{2} x, y$ ( $y$ is a 'reverse' of $\left.x\right), I^{1} x$ ( $x$ is an 'identity' arrow).

Arrow frames do not identify transitions with ordered pairs of states. Distinct arrows may have the same pair <input, output>, and not every such pair need have an arrow. Indeed, $C R S$-algebra suggests that arrows be ordered pairs, while giving up the idea that all ordered pairs are available. The resulting arrow frames need not be full Cartesian products of some state space. An even more radical version comes from category theory, with objects and morphisms. Let arrows be functions $f: A \rightarrow B$ inducing ordered pairs $\langle A, B>$ of 'source' and 'target'. Then, the relation $C$ expresses the partial function of composing maps, while reversal $R$ holds between a map and its inverse, if available. Quite different interpretations and applications may be found in Kurtonina 1995, which analyzes composition of linguistic expressions in the 'Lambek Calculus' of categorial grammar in arrow logic. Andréka \& Mikulas 1993 relate this back to relational algebra.

Language and truth definition Arrow frames $\boldsymbol{F}$ support a modal language that analyzes Relational Algebra. Arrow Models $\boldsymbol{M}$ add a propositional valuation $V$, and one can then interpret a matching modal propositional language defining properties of arrows using two modalities reflecting the basic 'ordering operations' of relational algebra: 


$$
\begin{array}{lll}
\boldsymbol{M}, x \mid=p & \text { iff } & x \in V(p) \\
\boldsymbol{M}, x \mid=\neg \phi & \text { iff } & \text { not } \boldsymbol{M}, x \mid=\phi \\
\boldsymbol{M}, x \mid=\phi \& \psi & \text { iff } & \boldsymbol{M}, x \mid=\phi \text { and } \boldsymbol{M}, x \mid=\psi \\
\boldsymbol{M}, x \mid=\phi \bullet \psi & \text { iff } & \text { there exist } y, z \text { with } C x, y z \text { and } \boldsymbol{M}, y|=\phi, \boldsymbol{M}, z|=\psi \\
\boldsymbol{M}, x \mid=\phi^{\vee} & \text { iff } & \text { there exists } y \text { with } R x, y \text { and } \boldsymbol{M}, y \mid=\phi \bullet \psi \\
\boldsymbol{M}, x \mid=I d & \text { iff } & \text { I } x
\end{array}
$$

Eventually, one can introduce more expressive modal operators into this vocabulary.

Modal logic The minimal logic of arrow models is an obvious counterpart of its monomodal version, whose key principles are the following axioms of Modal Distribution:

$$
\begin{array}{lll}
\left(\phi_{1} v \phi_{2}\right) \bullet \psi & \leftrightarrow & \left(\phi_{1} \bullet \psi\right) \vee\left(\phi_{2} \bullet \psi\right) \\
\phi \bullet\left(\psi_{1} v \psi_{2}\right) & \leftrightarrow & \left(\phi^{\bullet} \psi_{1}\right) \vee\left(\phi^{\bullet} \psi_{2}\right) \\
\left(\phi_{1} v \phi_{2}\right)^{\vee} & \leftrightarrow & \phi_{1} \vee v \phi_{2}
\end{array}
$$

A completeness theorem is provable here along standard lines, using Henkin models, with the usual techniques as explained, e.g., in Blackburn, de Rijke \& Venema 2000. The minimal logic is also decidable, again using a standard technique such as filtration.

\subsection{Arrow logic and relational algebra via modal correspondence}

Landscape of arrow axioms and frame correspondence On top of this minimal system, one can analyze axioms from Relational Algebra via constraints on arrow frames via frame correspondences. This analysis reveals a whole landscape of options. We only state results here, all of them follow by standard correspondence techniques using the 'Sahlqvist form' of the relevant axioms (cf. Blackburn, de Rijke \& Venema 2000):

Example Laws for Arrow Reversal.

$$
\begin{array}{ll}
\text { (1) } \quad \neg(\phi)^{2} \rightarrow(\neg \phi)^{\vee} \quad \text { iff } \quad \forall x \exists y R x, y \\
\text { (2) } \quad(\neg \phi)^{2} \rightarrow \neg(\phi)^{\vee} \quad \text { iff } \quad \forall x y z:(R x, y \& R x, z) \rightarrow y=z
\end{array}
$$

Together, these axioms make the binary relation $R$ a unary function $r$ of 'reversal'. Then the 'double conversion' axiom makes the function $r$ idempotent:

$$
(\phi)^{\sim} \leftrightarrow \phi \text { iff } \forall x r(r(x))=x
$$

With this notation, the following axioms connect reversal and composition: 
Example Arrow Composition Triangles.

(4) $\quad(\phi \bullet \psi)^{\vee} \rightarrow \psi^{\bullet} \phi^{\vee} \quad$ iff $\quad \forall x y z: C x, y z \rightarrow C r(x), r(z) r(y)$

(5) $\quad \phi \bullet \neg\left(\phi^{\bullet} \psi\right) \rightarrow \neg \psi \quad$ iff $\quad \forall x y z: C x, y z \rightarrow C z, r(y) x$

Together (2), (4), (5) imply the further interchange law $\forall x y z: C x, y z \rightarrow C y, x r(z)$.

Moreover, there is actually a more elegant form of axiom (5) without negation:

$$
\phi \&(\psi \bullet \chi) \rightarrow \psi \bullet(\chi \&(\psi \bullet \phi))
$$

Finally, the propositional constant $I d$ constrains 'identity loops'.

Example Identity Arrows.

(6) $\quad I d \rightarrow I d^{2} \quad$ iff $\quad \forall x: I x \rightarrow \operatorname{Ir}(x)$

(7) $\quad I d \bullet \phi \rightarrow \phi \quad$ iff $\quad \forall x y z:(I y \& C x, y z) \rightarrow x=z$

CRS-style core logic In our correspondence analysis of the basic axioms of Relational Algebra, some constraints come out as purely universal, making no demands on the supply of arrows. These seem the true core of action or computation. Universal frame constraints express laws for composition, converse and identity of arrows that lack existential import: by purely universal first-order sentences over arrow frames.

Fact The complete logic of arrow models satisfying all universal frame constraints valid in Relational Algebra is the set of validities for concrete $C R S$-style pair arrow models where arrows are ordered pairs - and the only change from standard models for relational set algebras is the limited supply of pairs.

This is easy to see. By contrast, existential constraints force the arrow set to become more like full Cartesian spaces, i.e., the standard models leading to undecidability.

Remark Associativity is existential.

One perhaps counter-intuitive feature of this analysis concerns Associativity for composition. Its frame condition is existential, requiring regroupings of transitions:

$$
\begin{array}{cl}
(\phi \bullet \psi) \bullet \chi \rightarrow \phi \bullet(\psi \bullet \chi) \quad \text { iff } \quad & \forall x y z u v:(C x, y z \& C y, u v) \rightarrow \\
& \exists w:(C x, u w \& C w, v z))
\end{array}
$$

(8b) and likewise in the opposite direction. 
Now Associativity is often considered a useful core feature of dynamic logics: different orders of composition are equivalent. In contrast, $C R S$-style arrow logic distinguishes different ways of 'chunking' parts, while Associativity can lead to undecidability. Similar points are known from categorial logics (van Benthem 1996B, Chapter 12).

\subsection{Arrow logic over pair models}

Axiomatics Here is a complete axiomatization for the logic of pair arrow models with the obvious definitions for composition, reversal and identity (Marx 1995):

Theorem The following set of principles is complete for pair arrow models:

(1) the minimal arrow logic, (2) converse is an idempotent function, (3) identity laws $I d \leftrightarrow I d \bullet I d, I d \leftrightarrow I d^{\llcorner}, \phi \& i d \rightarrow \phi^{\llcorner}, I d \bullet \phi \leftrightarrow \phi, \phi \bullet I d \leftrightarrow \phi$, $\phi \bullet \neg \phi^{\llcorner} \rightarrow \neg I d$, (4) 'triangle laws' $\phi \bullet \neg\left(\phi^{\vee} \psi\right) \rightarrow \neg \psi, \neg\left(\phi \bullet \psi^{\llcorner}\right) \bullet \psi \rightarrow \neg \phi$,

(5) the limited associativity principles $((\phi \& I d) \bullet \psi) \bullet \chi \leftrightarrow(\phi \& I d) \bullet(\psi \bullet \chi)$, $((\phi \bullet(\psi \& I d)) \bullet \chi \leftrightarrow \phi \bullet((\psi \& I d) \bullet \chi),(\phi \bullet \psi) \bullet(\chi \& I d) \leftrightarrow \phi \bullet(\psi \bullet(\chi \& I d))$.

Further pleasant properties of this system include decidability and Craig interpolation. Many further results are in Marx 1995, Mikulas 1995, while Marx \& Venema 1997 is an excellent general source, also referencing the seminal work of the Budapest group.

Language extensions: dynamic arrow logic Pair arrow logic retains its nice properties when we extend its vocabulary with a universal modality, or other operators. These moves exemplify a program of trading deductive strength for expressive power. One important extension yields Dynamic Arrow Logic, with an infinitary operator for composition similar to the crucial Kleene iteration of propositional dynamic logic:

$$
\begin{gathered}
M, x \mid=\phi^{*} \quad \text { iff } \quad x \text { can be } \text { C-decomposed into some finite sequence } \\
\text { of arrows satisfying } \phi \text { in } \boldsymbol{M} .
\end{gathered}
$$

Defined in this way, $\phi^{*}$ satisfies the following simple laws that yield the system $D A L$ :

(9) $\phi \rightarrow \phi^{*}$

(10) $\phi^{*} \cdot \phi^{*} \rightarrow \phi^{*}$

(11) if $\mathrm{I}-\phi \rightarrow \alpha$ and $\mathrm{I}-\alpha \bullet \alpha \rightarrow \alpha$, then $\mathrm{I}-\phi^{*} \rightarrow \alpha$

Theorem (van Benthem 1994) DAL is complete for its intended interpretation. 
Remark: dynamic logic as two-sorted arrow logic. One can mirror dynamic logic more closely by combining arrow logic with a modal logic of states. This involves operators of test ? from propositions to programs, and domain $\diamond$ from programs to propositions. These are non-homogeneous distributive modalities correlating states and arrows:

$$
\begin{array}{ll}
\boldsymbol{M}, x \mid=\phi ? & \text { iff } \quad \text { there exists some } s \text { with } T x, s \text { and } \boldsymbol{M}, s \mid=\phi \\
\boldsymbol{M}, x \mid=<\pi> & \text { iff } \quad \text { there exists some } x \text { with } D s, x \text { and } \boldsymbol{M}, x \mid=\pi
\end{array}
$$

Intuitively, the relation $T x$, says that $x$ is an identity arrow for the point $s$, while $D s, x$ says that $s$ is a left end-point of the arrow $x$. Via frame correspondences, axioms on?, $\diamond$ impose special connections between $T$ and $D$. For instance, $\langle\phi$ ? $>\leftrightarrow \phi$ expresses the conjunction of $\forall s \exists x: D s, x \& T x$, $s$ and $\forall s x: D s, x \rightarrow \forall s^{\prime}: T x, s^{\prime} \rightarrow s=s^{\prime}$.

\subsection{A fragment view?}

In Section 1, we claimed that generalized semantics may sometimes be reinterpreted as a move to restricted syntax. Indeed, Arrow Logic or $C R S$-versions of relational algebra, may be translated into fragments of first-order logic involving guarded quantification. But we will explain this connection only later on, in Section 4 below.

\section{From $C R S$ to general assignment models for first-order logic}

\subsection{The core mechanics of first-order semantics, a modal perspective}

The main recursion The standard semantics for predicate logic has this key clause:

$$
\boldsymbol{M}, \alpha \mid=\exists x \phi \text { iff for some } d \in|\boldsymbol{M}|: \boldsymbol{M}, \alpha^{x} d=\phi
$$

The key is the use of variable assignments $\alpha$ that decompose quantified statements with free variables in their matrix. But looking at the usual truth definition, a compositional semantics for first-order quantification only needs the following abstract core pattern:

$$
\boldsymbol{M}, \alpha \mid=\exists x \phi \quad \text { iff } \quad \text { for some } \beta: R_{x} \alpha \beta \text { and } \boldsymbol{M}, \beta \mid=\phi
$$

Here, assignments $\alpha, \beta$ become abstract states, and the concrete relation $\alpha={ }_{x} \beta$ - which held between $\alpha$ and $\alpha^{x}$-become just any binary relation $R_{x}$. This involves poly-modal models $\boldsymbol{M}=\left(S,\left\{R_{x}\right\}_{x \in V A R}, I\right)$ with $S$ a set of states, $R_{x}$ a binary update relation for each variable $x$, and $I$ an interpretation function giving truth values to atomic formulas $P x$, $R x y, \ldots$ in each state $\alpha$. Thus existential quantifiers $\exists x$ become existential modalities 
$<x>$. This abstract semantics has an independent appeal: first-order evaluation is an informational process that changes computational states, as in the 'dynamic semantics' of Groenendijk \& Stokhof 1991, Veltman 1996. First-order logic then becomes a dynamic logic with a special choice of atoms and no explicit compound programs.

'Deconstruction' From this modal point of view, standard semantics arises by insisting on three additional mathematical choices, not enforced by the core semantics:

(a) States are identified with variable assignments,

(b) Update between states must be the specific relation $=_{x}$, and

(c) All assignments in the function space $D^{V A R}$ are available to evaluation.

The former are issues of implementation, the latter a strong existence assumption. (Actually, standard first-order logic needs only locally finite assignments.) Henceforth, we shall regard these further choices as negotiable. In fact, it is often felt that tricks like making predicates sets of tuples should be orthogonal to the nature of logical validity.

Minimal logic Our modal semantics validates the minimal poly-modal logic with

- $\quad$ all classical Boolean propositional laws

- $\quad$ Modal Distribution: $\forall x(\phi \rightarrow \psi) \rightarrow(\forall x \phi \rightarrow \forall x \psi)$

- Modal Necessitation: if $\mid-\phi$, then $\mid-\forall x \phi$

- $\quad$ a definition of $\exists x \phi$ as $\neg \forall x \neg \phi$

This logic is complete, and has the usual properties of first-order logic, such as Craig interpolation or the Los-Tarski preservation theorem. One can now usefully pursue standard first-order model theory in tandem with its modal counterpart. For instance, consider modal bisimulations for these models, relating states making the same atoms true, with zigzag conditions for the relations $R_{x}$. Specializing these to standard models leads to the standard notion of potential isomorphism (de Rijke 1993, van Benthem 1996B, van Benthem \& Bonnay 2008). And in all this, the modal system is decidable.

Landscapism The modal perspective suggests a landscape below standard predicate logic, with a minimal modal logic at the base, ascending to standard semantics via frame constraints. In particular, this landscape contains decidable sublogics of predicate logic, sharing its desirable meta-properties. Thus, the 'undecidability of predicate logic' largely reflects mathematical accidents of its Tarskian modeling, encoding set-theoretic 
facts about function spaces $D^{V A R}$ - beyond the core logic of quantification and variable assignment. We shall explore this view of first-order semantics, including richer languages. Abstract core models support new distinctions between various forms of quantification ('monadic' and 'polyadic') that get collapsed in standard predicate logic.

\subsection{From modal state models to general assignment models}

To recapitulate, we have just re-interpreted first-order logic as a modal logic on a much more general class of abstract modal state models

$$
\boldsymbol{M}=\left(S,\left\{R_{x}\right\} x \in V A R, I\right)
$$

with $S$ a set of 'states', $R_{x}$ a binary accessibility relation between states for each variable $x$, and $I$ an interpretation function giving a truth value to each atomic formula in each state $s$. This is a huge extension of standard semantics, where no domain of 'individual objects' need now be present underpinning the states. Quantifiers became modalities:

$$
\boldsymbol{M}, s \quad \mid=\exists x \phi \quad \text { iff } \quad \text { for some } t: R_{X} \text { st and } \boldsymbol{M}, t \mid=\phi .
$$

More concrete is the following halfway house, an intermediate semantics that retains assignments as the state space - just taking away the existential assumption of 'fullness' from standard Tarski models for first-order logic.

Definition General assignment models.

A general assignment model is an ordered pair $(\boldsymbol{M}, \boldsymbol{V})$ with $\boldsymbol{M}$ a standard first-order model with domain $D$ and interpretation function $I$, and $V$ any non-empty set of assignments on $\boldsymbol{M}$, i.e., a subset of $D^{V A R}$. The first-order language is interpreted as usual, now at triples $\boldsymbol{M}, V, s$ with $s \in \mathbb{V}$ - with the following clause for quantifiers:

$$
\boldsymbol{M}, V, s \mid=\exists x \phi \text { iff for some } t \in V: s={ }_{x} t \text { and } \boldsymbol{M}, V, t \mid=\phi
$$

Here $=x$ is the standard relation between assignments of identity up to $x$-values.

Remark: independent motivations The 'gaps' in general assignment models are not just a trick for lowering complexity: they may be seen as modeling the natural phenomenon of dependencies between variables. Changes in value for one variable $x$ may induce, or at least be correlated with, changes in value for another variable $y$. Hintikka \& Sandu 1997, Hodges 2001, Väänänen 2007 are other recent approaches to dependence in logic. 
Also, van Benthem \& Martinez 2008 point out how the above modal perspective on dependence given here may also be related to the analysis of constraint-based information flow in situation semantics (cf. Barwise \& Seligman 1995, Mares 2003).

\subsection{Complete base logic}

The complete set of validities for the new semantics is still well-behaved:

Theorem The logic of general assignment models is completely axiomatized by the standard axioms for the poly-modal version of the logic $S 5$ plus all atomic 'locality principles' $(\neg) P \boldsymbol{x} \rightarrow \forall \boldsymbol{y}(\neg) P \boldsymbol{x}$ with $\boldsymbol{x} \cap \boldsymbol{y}=\varnothing$.

This complete logic 'CRS' has been much studied (excellent sources are Németi 1995, Marx \& Venema 1997). The completeness proof involves various representation arguments. Some results from van Benthem 1996B, Sections 9.8 and 9.9, give the flavour. Let $\boldsymbol{x}, \boldsymbol{y}$ be finite sequences of variables. The notation $R_{x}$ denotes the sequential composition of accessibility relations $R_{x}$ as they occur in their given order in $\boldsymbol{x}$.

Theorem An abstract modal frame $\left(S,\left\{R_{x}\right\} x \in V A R\right)$ is isomorphic to the frame of a general assignment model iff the $R_{x}$ are equivalence relations satisfying two 'Path Principles' (a) if $s R_{\boldsymbol{z} l} t, \ldots, s R_{\boldsymbol{Z} k} t$, and the only variable occurring in all $z_{1}, \ldots, z_{k}$ is $x$, then $s R_{x} t$, (b) if no variable occurs in all of $z_{1}, \ldots, z_{k}$, then $s=t$.

Less is needed if we are content with a weaker equivalence than isomorphism:

Theorem A finite modal model is bisimilar to a general assignment model if and only if its accessibilities are all equivalence relations.

Typically not universally valid in arbitrary modal models are the following principles:

$$
\begin{array}{llll}
\text { (i) } & {[\boldsymbol{u} / \boldsymbol{y}] \psi \rightarrow \exists y \psi} & \text { with } \boldsymbol{u} \text { free for } \boldsymbol{y} \text { in } \psi & \text { Existential Generalization } \\
\text { (ii) } & \phi(x) \rightarrow \forall \boldsymbol{y} \phi(\boldsymbol{x}) & \text { with no } \boldsymbol{y} \text { free in } \phi(x) & \text { Full Locality }
\end{array}
$$

Viewed positively again, these failures reflect the special handling of variables in models where not all assignments need be available. All of $x, y, z, \ldots$ then acquire a sort of 'individuality', due to interactions with other variables. As we said earlier, variables can now have or lack dependencies, which again gives them a certain individuality.

$C R S$ is also decidable, using modal filtration techniques. We omit details here. 


\subsection{Language extensions}

General assignment models do not just make first-order logic weaker. They also support an enrichment, in supporting new vocabulary reflecting distinctions that could not yet be seen in standard first-order logic. Examples are irreducibly polyadic quantifiers $\exists \boldsymbol{x}$ binding tuples of variables $\boldsymbol{x}$, with the following truth condition:

$M, V, s \mid=\exists x \phi$ iff for some $t \in V: s={ }_{x} t$ and $M, V, t \mid=\phi$

This time, $=\boldsymbol{x}$ is identity between assignments up to values for all the variables in $\boldsymbol{x}$. In standard first-order logic, the notation $\exists x y \bullet \phi$ is just short-hand for $\exists x \exists y \phi$ or $\exists y \exists x \phi$ in any order. But in $G A M$-semantics, these two expressions are no longer equivalent, as not all 'intermediate assignments' for $x$ - or $y$-shifts need be present - and they are both non-equivalent to $\exists x y$, as defined just now. Moreover, one can also interpret single or polyadic substitution operators directly in this style (cf. Venema 1994):

$$
\boldsymbol{M}, V, s \mid=[\boldsymbol{y} / \boldsymbol{x}] \phi \text { iff } s[\boldsymbol{x}:=s(\boldsymbol{y})] \in V \& \boldsymbol{M}, V, s[\boldsymbol{x}:=s(\boldsymbol{y})] \mid=\phi
$$

\subsection{General semantics for non-first-order fixed-point languages}

General assignment models also suggest new perspectives on non-first-order systems, in particular fixed-point logics of computation and action in general. Consider the fixedpoint version $\operatorname{LFP}(F O)$ of first-order logic (Ebbinghaus \& Flum 1995). This language extends the usual inductive formation rules for first-order syntax with an operator

$$
\mu P, x \bullet \phi(P, Q, x)
$$

where $P$ may occur only positively in $\phi(P, \boldsymbol{Q}, \boldsymbol{x})$, and $\boldsymbol{x}$ is a tuple of variables of the right arity for $P$. The relevant predicates are the smallest fixed-points of the following monotone set operation on predicates in any given model $\boldsymbol{M}$ :

$$
F_{\phi}^{M}=\lambda P \bullet\{\boldsymbol{d} \text { in } M|(\boldsymbol{M}, P), \boldsymbol{d}|=\phi(P, \boldsymbol{Q})\}
$$

With the fixed-point theorems underpinning this system, we see a process of successive approximation for the predicate $P$ that involves changing assignments through ordinal stages. In this process, the full space $D^{V A R}$ is usually taken for granted, may depend on the available assignments, and thus, in our present terms, the 'relativized' version of $L F P(F O)$ is worth exploring. We make a few observations (cf. van Benthem 2005). 
The syntax for formulas $\phi$ in the language $\operatorname{LFP}(F O)$ now needs a bit more care, since variables are less 'anonymous' in general assignment models, as we noted before. In particular, when defining a predicate $\mu P, \boldsymbol{x} \bullet \phi(P, \boldsymbol{Q}, \boldsymbol{x})$, the particular variables $\boldsymbol{x}$ matter. This suggests we are only defining values for the specific atom $P \boldsymbol{x}$, whereas variants such as $P \boldsymbol{y}$ must be viewed as substitution instances $[\boldsymbol{y} / \boldsymbol{x}] P \boldsymbol{x}$. With this understanding, we can give a definition of semantic evaluation as before.

Definition GAM fixed-point evaluation.

Formulas $\phi$ in the above language induce the following map in general assignment models $(\boldsymbol{M}, V)$ with some given assignment $s$ for the free variables in $\phi$ :

$$
F_{\phi}^{M, s}=\lambda P \bullet\{\boldsymbol{d} \text { in } M|s[\boldsymbol{x}:=\boldsymbol{d}] \in V \&(\boldsymbol{M}, P), s[\boldsymbol{x}:=\boldsymbol{d}]|=\phi(P, Q)\}
$$

Smallest and greatest fixed-points are then defined as usual.

Example Transitive closure of guard predicates.

Consider the fixed-point formula $\phi=\mu P, x \bullet Q x \vee \exists y[y / x] P x$. Its approximation sequence as defined above starts with the empty set for $P$, and it ends by stage $\omega$, where iteration of the map $F_{\phi}^{M, s}$ produces nothing new. Here are some stages:

$$
\begin{aligned}
& P^{0} \quad=\varnothing \\
& P^{l} \quad=\quad\left\{d\left|s[x:=d] \in V \&\left(\boldsymbol{M}, P^{0}\right), s[x:=d]\right|=Q x \vee \exists y[y / x] P x\right\} \\
& =\quad\{d \mid s[x:=d] \in V \& Q(d)\} \\
& P^{2} \quad=\quad\left\{d\left|s[x:=d] \in V \&\left(\boldsymbol{M}, P^{l}\right), s[x:=d]\right|=Q x \vee \exists y[y / x] P x\right\} \\
& =\{d \mid s[x:=d] \in V \&(Q(d) \vee \text { for some object e }: \\
& s[x:=d][y:=e] \in V \& s[x:=e][y:=e] \in V \& Q(e))\}
\end{aligned}
$$

Iteratively, one computes the set of all objects $d$ for which there is an object $e$ satisfying $Q$ reachable from $d$ in the transitive closure of the following relation:

$$
R_{s} a b \quad \text { iff } \quad s[x:=a][y:=b], s[x:=b][y:=b] \in V .
$$

We forego further details here - but note how these fixed-point computations bring to light the hidden dependency structure of the relevant general model.

Theorem $\quad \operatorname{LFP}(F O)$ is decidable over general assignment models. 
Proof This follows from Grädel's result on the decidability of the fixed-point version $L F P(G F)$ of the Guarded Fragment in Section 4 below. The translation guard given there from arbitrary formulas to guarded ones easily extends to a language with added fixed-point operators. Next, translations are inside the language of $L F P(G F)$, and fixedpoint evaluation must stay inside the set of tuples satisfying the guard relation $R$.

What this brief exploration suggests are far more general uses for general assignment models in the abstract model theory for arbitrary extensions of first-order logic.

\section{$4 \quad$ Basics of the Guarded Fragment}

Next, we look at the other way of importing relativization into logic, through the syntax of suitably chosen fragments of standard logical languages. The system that follows arose from a combination of two sources. One was cylindric relativized set algebra and its generalized models for first-order logic, the other reflection on what makes modal logic tick as a source of decidable well-behaved fragments of first-order logic (van Benthem 1995). The two ways of thinking came together in the following large sublanguage of first-order logic, which again has an independent intuitive motivation.

\subsection{Guarded syntax}

The Guarded Fragment of Andréka, van Benthem \& Németi 1998 is a decidable part of first-order syntax with a semantic philosophy: quantifiers only access the total domain of individual objects 'locally' by means of predicates over objects. But there is more to the ambitions of guarding as a method, as will become clear in due course.

Here are some syntactic preliminaries. In what follows, mostly for convenience, we consider only first-order languages with predicate symbols and variables: no function symbols or identity predicates occur. But we do allow polyadic quantifiers $\exists x \phi, \forall x \phi$ over tuples of variables $\boldsymbol{x}$, with their obvious interpretation, which resembles the polyadic quantifiers discussed earlier in connection with general assignment models. Even though we can rewrite these in terms of successive single first-order quantifiers, we may not be able to do so inside the fragments we are studying. We also use polyadic simultaneous substitutions $[\boldsymbol{u} / \boldsymbol{y}] \phi$ that need not reduce to iterated single substitutions. These are taken in the standard syntactic sense that substitution is performed provided the $\boldsymbol{u}$ are free for the $\boldsymbol{y}$. If not, some suitable alphabetic variant is taken first for $\phi$. 
Our key idea is that objects $\boldsymbol{y}$ can only be introduced relative to given objects $\boldsymbol{x}$, as expressed by a 'guard atom' $G(\boldsymbol{x}, \boldsymbol{y})$ where objects can occur in any order and multiplicity - and that the subsequent statement refers only to those guarded $\boldsymbol{x}, \boldsymbol{y}$.

Definition Guarded Formulas.

Guarded formulas are all those constructed according to the syntax rules

$$
\text { atoms } P \boldsymbol{x}|\neg| \vee \mid \exists y(G(x, y) \& \phi(x, y))
$$

Here, bold-face $\boldsymbol{x}, \boldsymbol{y}$ indicate finite tuples of variables, and $G$ is a predicate letter.

Clearly, the well-known 'standard translation' takes basic modal logic into first-order formulas lying inside the Guarded Fragment, and the same is true for many other modal languages in the literature. For further illustrations and applications, see van Benthem 2001, which also considers guarded patterns in modal neighbourhood semantics.

\subsection{Decidability of GF via quasi-models}

The initial motivating result was that guarding quantifiers leads to decidability.

Theorem $\quad G F$ is decidable.

The proof of Theorem 1 is worth stating here in outline, for the general ideas involved. Proof The first observation is that truth of first-order formulas in any model is witnessed in some finite syntactic object, called a 'quasi-model'. Let formula $\phi$ be true in standard model $\boldsymbol{M}$. Let $V$ be the finite set of variables occurring in $\phi-$ free or bound. In effect, we are inside a finite-variable fragment of first-order logic here. Next, we restrict attention to the finite set $\operatorname{Sub}_{\phi}$ consisting of $\phi$ and its sub-formulas, while also closing under simultaneous substitutions $[\boldsymbol{u} / \boldsymbol{y}]$ using only variables in $V$, that do not change syntactic forms. This is feasible because of the following simple observation, provable by some syntactic manipulation:

Lemma Finite-variable fragments are closed under simultaneous substitutions.

Now each variable assignment $s$ on $\boldsymbol{M}$ verifies a set $\Delta_{s}$ of formulas from $S u b_{\phi}$ with special properties, that we call a type. Note that any model realizes at most finitely many types. A 'quasi-model' is a finite set of types with some properties and mutual relations that obviously hold if the source is indeed some model $\boldsymbol{M}$. 
Definition Quasi-models.

Let $F$ be the finite set of all formulas of length $\leq|\phi|$ that use only variables from $V$. Note that $\phi \in F$ and $F$ is closed under taking sub-formulas and the above 'alphabetic variants' used with substitutions. An F-type is a subset $\Delta$ of $F$ which satisfies
(a) $\neg \psi \in \Delta$
iff
not $\psi \in \Delta$
whenever $\neg \psi \in F$
(b) $\psi v \xi \in \Delta$
iff
$\psi \in \Delta$ or $\xi \in \Delta$
whenever $\psi v \xi \in F$
(c) $[\boldsymbol{u} / \boldsymbol{y}] \psi \in \Delta$
only if
$\exists y \psi \in \Delta$
whenever $\exists y \psi \in F$

Next, we write $\Delta=\boldsymbol{y} \Delta^{\prime}$ if $\Delta, \Delta^{\prime}$ share the same formulas with all their free variables disjoint from $y$. A quasi-model is a set of $F$-types $S$ such that

(d) for each $\Delta \in S$ and each formula $\exists y \psi \in \Delta$,

there is a type $\Delta^{\prime} \in S$ with $\psi \in \Delta^{\prime}$ and $\Delta=y \Delta^{\prime}$.

We say that $\phi$ holds in a quasi-model if $\phi \in \Delta$ for some $\Delta$ in this quasi-model.

Clearly, this definition justifies the following assertion:

Lemma If a first-order formula has a model, it is true in some quasi-model.

The converse is not true for all first-order formulas, but it does hold for $G F$.

Lemma If a guarded formula has a quasi-model, then it has a standard model.

The key fact is that quasi-models can be 'unraveled' to tree-like standard models without affecting truth values of guarded formulas in their set $F$ : details of the proof are in Andréka, van Benthem \& Németi 1998. Decidability of $G F$ now follows because we can test satisfiability for arbitrary (loosely) guarded formulas $\phi$ by testing for the existence of a quasi-model for $\phi$ whose size is effectively bounded by the length of $\phi$.

This decision procedure can be adapted easily to give an optimal complexity result (Grädel 1999B). Satisfiability is 2EXPTIME-complete for guarded formulas, and it is EXPTIME-complete for $G F$ with a fixed bound on the arities of predicates.

\subsection{Other meta-properties}

The Guarded Fragment was meant to serve several purposes at once. On the one hand its complexity is low enough to be decidable, while it is expressive enough to generalize 
most common modal languages. This demonstrates the balance sought in all good modal-like languages. Another desirable feature concerns its meta-theory.

Basic modal logic resembles first-order logic in all its meta-properties, partly thanks to its having a generalization of modal bisimulation. Let a partial isomorphism be a finite one-to-one partial map between models which preserves relations both ways. In any model $\boldsymbol{M}$, call a set $X$ of objects guarded if there is a relation symbol $R$, say $k$-ary, and objects $a_{l}, \ldots, a_{k} \in M$ (possibly with repetitions) with $R^{M_{(}}\left(a_{1}, \ldots, a_{k}\right)$ and $X=\left\{a_{1}, \ldots, a_{k}\right\}$.

\section{Definition Guarded Bisimulations.}

A guarded bisimulation is a non-empty set $\boldsymbol{F}$ of finite partial isomorphisms between two models $\boldsymbol{M}$ and $\boldsymbol{N}$ that satisfies the following two back-and-forth conditions for any $f: X \rightarrow Y$ in $\boldsymbol{F}$ : (i) for any guarded $Z \subseteq M$, there is a $g \in \boldsymbol{F}$ with domain $Z$ such that $g$ and $f$ agree on the intersection $X \cap Z$, (ii) for any guarded $W \subseteq \mathrm{N}$, there is a $g \in \boldsymbol{F}$ with range $W$ such that the inverses $g^{-1}$ and $f^{-1}$ agree on $Y \cap W$.

The point of this definition shows in semantic invariance for guarded bisimulation:

Fact Let $\boldsymbol{F}$ be a guarded bisimulation between models $\boldsymbol{M}$ and $\boldsymbol{N}$ with $f \in \boldsymbol{F}$.

For all guarded formulas $\phi$ and variable assignments $\alpha$ into the domain of $f$, we have that $\boldsymbol{M}, \alpha \mid=\phi$ iff $\boldsymbol{N}$, fo $\alpha \mid=\phi$.

The following result closely follows an analogue for modal logic and bisimulation.

Theorem Let $\phi$ be any first-order formula. The following two assertions are equivalent:

(a) $\phi$ is invariant for guarded bisimulations, (b) $\phi$ is equivalent to a $G F$ formula.

Techniques based on this invariance establish even meta-properties of $G F$ that do not follow from just being a sublanguage of first-order logic, such as Craig Interpolation, Beth Definability, and the standard model-theoretic preservation theorems. GF shares this good behaviour to a large extent, witness the Los-Tarski theorem for $G F$-formulas that are preserved under taking sub-models given in Andréka, van Benthem \& Németi 1998. Cf. also van Benthem 2001 on $G F$ as an instrument for finding out 'what makes modal logic tick'. But subsequent work has shown that the picture is somewhat mixed:

Theorem Beth Definability holds but Craig Interpolation fails for $G F$. Interpolation remains valid when we view guard predicates as part of the logical vocabulary.

Proofs are in Hoogland, Marx \& Otto 1999, and in Hoogland \& Marx 2002. 


\subsection{Excursion: quasi-models per se}

The methods around GF may have a broader spin-off. In particular, quasi-models are a mix of modal filtration, semantic tableaus for first-order logic, and the 'mosaics' of algebraic logic. Right now, mosaics - introduced in Németi's 1986 dissertation, with Németi 1995 as a more up-to-date reference - seem the method of choice for proving decidability in modal and algebraic logics. But quasi-models may also be appreciated on their own. First, a quasi-model for some initial formula $\phi$ is a modal model $\boldsymbol{M}_{\phi}$ for a first-order language as it stands. The types are the worlds, there are accessibility relations $=_{x}$ of agreeing on all formulas having no free variables in $\boldsymbol{x}$, and for atoms, $V(\Delta, P \boldsymbol{x})=1$ iff $P \boldsymbol{x} \in \Delta$. The following Truth Lemma is then easily proved by induction:

Lemma For all $\alpha \in S U B_{\phi}$, and all types $\Delta$ in $\boldsymbol{M}_{\phi}, \mathbf{M}, \Delta \mathrm{l}=\alpha$ iff $\alpha \in \Delta$.

Thus, quasi-models are models by themselves, and this may lead to new links between modal and first-order logic. Modal models may be related to quasi-models modulo forms of bisimulation (van Benthem 1996B). Still, having a quasi-model - finite by definition - does not imply having a finite standard model. It is easy to find a quasimodel for the formula $\forall x y z((R x y \& R y z) \rightarrow R x z) \& \forall x \exists y R x y \& \forall x \neg R x x$ which only has infinite models. In fact, having a quasi-model need not imply standard satisfiability at all. The predicate-logically inconsistent formula $\exists x \exists y$ Rxy \& $\neg \exists y$ $\exists x$ Ry is clearly satisfiable in the general assignment model $\boldsymbol{M}$ with domain $\{1,2\}$, relation $\{<1,2>\}$, and just one admissible assignment $s$, viz. $\{(x, 1),(y, 2)\}$. This model also satisfies the earlier Existential Generalization and Full Locality. The single type of $\boldsymbol{M}$ induced by $s$ is therefore a quasi-model for $\exists x \exists y R x y \& \neg \exists y \exists x R x y$. This 'inconsistency' in a set of types may seem strange - but it also shows that quasi-models are intriguing structures.

\subsection{Extensions}

$G F$ is not yet the end of the road. Analyzing the earlier proof of decidability, van Benthem 1997A noticed that it goes through for the following extension.

Definition Loosely guarded formulas extend the syntax of $G F$ by allowing a conjunction of atoms $\gamma(\boldsymbol{x}, \boldsymbol{y})$ instead of $G(\boldsymbol{x}, \boldsymbol{y})$ in the quantifier clause, provided each variable from $\boldsymbol{y}$ co-occurs with each variable from $\boldsymbol{x}, \boldsymbol{y}$ in at least one atom of $\gamma(\boldsymbol{x}, \boldsymbol{y})$. This yields the Loosely Guarded Fragment LGF. 
The point here is that the guarding of objects does not take place all at once, but twoby-two. This suffices for several earlier results:

Theorem Both $L G F$ and its fixed-point extension are decidable.

As an application, modal logics like that of temporal Since and Until are decidable, since their truth conditions are typically loosely guarded (van Benthem 1997A, 2001). For instance, $U N T I L p q$ is defined by the $L G F$-formula

$$
\exists x<y \& \forall z((x<z \wedge z<y) \rightarrow Q z)) .
$$

Another striking application is the decidability of the earlier complete logic of pair arrow models. The translation in Section 2 that relativized to the 'top relation' $U$ does not take formulas ' $(x, y): R$ ' into $G F$ itself, but it does take them into $L G F$ !

Next, by way of contrast, consider the first-order property of transitivity, which can lead to undecidable fragments of $F O L$. It has 'one guard too few':

$$
\forall x y z((x<z \wedge z<y) \rightarrow x<y))
$$

Next, consider non-first-order extensions, like we had before in Sections 2, 3. A striking positive result concerns the extension $L F P(G F)$ of $G F$ with fixed-point operators $\mu, v$ :

Theorem (Grädel 1999C) $\quad L F P(G F)$ is decidable.

By contrast, validity for the fixed-point extension $L F P(F O)$ of full first-order logic is of high non-arithmetical complexity, as it can define the natural numbers categorically. Grädel 1999A also determines the computational complexity for $\operatorname{LFP}(G F)$.

As a small application, the preceding result explains the validity of many modal logics over transitive models, even though transitivity by itself is dangerously non-guarded. Instead of working over transitive models, take models with arbitrary binary relations. Then a modality for a special transitive relation becomes an iteration modality for the transitive closure of the given arbitrary relation, which can be defined inside $L F P(G F)$.

\subsection{Border line: confluence}

A useful alternative way of understanding the guarding technique looks 'from above'. What expressive resources will typically lead to un-decidability? Here is a natural comparison with a related, though subtly different fragment of first-order logic. 
Bounding versus guarding Bounded formulas have all their quantifiers relativized to an atomic predicate, as in the ubiquitous pattern $\exists x: x \in y$ in set theory. Feferman \& Kreisel 1969 show that the characteristic semantic feature of bounded first-order formulas is their invariance for generated sub-models. The Bounded Fragment BF differs from the Guarded Fragment in allowing the more general format of quantification

$$
\exists y(G(x, y) \& \phi(x, y, z))
$$

where the formula at the end may contain new free variables. $B F$ is undecidable, but it has applications in arithmetic and set theory, as a way of defining 'absolute' properties not affected by the difference between standard models and generalized models. Ten Cate 2005 has a modern treatment with new results, including the one that $B F$ equals the first-order definable part of basic modal logic with added propositional quantifiers. Finally, van Benthem 2005 shows how, following Montague 1970, Gallin 1975, bounding serves as a general technique for lowering complexity in second-order logic.

Tiling problems, grid structure, and confluence One way of seeing that $B F$ is undecidable is by noting that it can define the following geometrical Tiling Problem. Let a finite set of 'tiles' be given, with colours on each of their four sides. 'Matching tiles' have the same colours on adjacent sides. Now the Tiling Problem asks:

Can we cover the whole plane $N \times N$ with matching tiles from the given set?

Fact The Tiling Problem is undecidable.

Tiling has the same complexity as the Non-Halting Problem: deciding if a given Turing machine will keep computing forever on given input. (To see the $\Pi_{1}^{0}$-complexity, note that one can tile a plane iff one can tile all finite sub-planes, and use Koenig's Lemma.) Indeed, the two problems are equivalent, as one can code successive tape contents as horizontal rows, with the vertical sequence as the 'computation'. It is essential here that positions on successive rows can be compared in the right way, and this is what the grid structure of $N x N$ does. Since tiling problems are easier to encode than Turing machine computations, they have gained popularity as a way of showing high complexity.

Now it is easy to show the undecidability of first-order logic. Consider a set $T$ of square tiles $\left\{t_{1}, \ldots, t_{m}\right\}, T P$ is the task of putting one tile on each point in the grid $N x N$ giving 
adjacent edges the same colour. We can reduce this task effectively to a satisfiability problem in first-order logic, by constructing a formula $\phi_{T}$ with the following property:

Fact $\phi_{T}$ is satisfiable iff the given set $T$ can tile the INxIN-plane.

The formula $\phi_{T}$ is constructed as follows. We choose unary predicates $P_{t}$ for each tile $t$, and binary relations NORTH and EAST for moving around in the grid, whose one-step immediate successor versions are $\mathrm{NORTH}^{+}, \mathrm{EAST}^{+}$. Now we write up what tiling amounts to. Note that the 'adjacent colours' condition just amount to giving an ordering from tiles to a finite set of 'fitting tiles' in each direction. More concretely, each point then has to satisfy the following properties, involving only finite formulas in total:

(a) the exhaustive finite disjunction $T$ of all tiling predicates $P_{t}$,

(b) $\left(P_{t} x \wedge N O R T H^{+} x y\right) \rightarrow$ 'disjunction of all $P_{t}$ ' with $t$ ' fitting to the north'(y), $\left(P_{t} x \wedge E A S T^{+} x y\right) \rightarrow$ 'disjunction of all $P_{t}$ ' with $t$ ' fitting to the east' $(y)$,

(c) NORTH, EAST are transitive irreflexive relations with successors,

(d) $\forall x y z:\left(N O R T H^{+} x y \wedge E A S T^{+} y z\right) \rightarrow \exists u:\left(E A S T^{+} x u \wedge N O R T H^{+} u z\right)$,

(e) there is a unique initial point for the whole structure.

We prefix a universal quantifier to make sure it holds everywhere in the model.

It is clear how to satisfy $\phi_{T}$ given a tiling. But also conversely, if $\phi_{T}$ is satisfiable at some point $s$ in a grid-like model $\boldsymbol{M}$, we can use the unary predicates $P_{t}$ in $\boldsymbol{M}$ to tile the plane IN $x I N$. Working from the origin, first read off the tiling for the initial point, and then proceeding inductively, tile in triangles, using the grid property of the model to place the next edge in such a way that no conflicts arise in the placement pattern.

The preceding reduction shows that satisfiability for first-order logic is undecidable, since the Tiling Problem is. Now, the crucial feature behind this reduction is the grid structure, defined by the confluence property (d). While this formula employs bounded quantifiers, putting it in $B F$, it is typically not in $G F$ or even $L G F$ : not all pairs of objects come with an atomic bound, witness the case of $y$ and $z$. This is significant. Grid structure tends to involve high complexity, a fact also known from modal logics where combining two modalities for two relations which satisfy a commutation axiom expressing grid structure (Halpern \& Vardi 1989, van Benthem \& Pacuit 2006). 
Remark Trees versus Grids.

By contrast, many ordinary modal logics are decidable since their semantics is based on trees rather than grids, and then we are in the realm of Rabin's Theorem saying that the complete monadic second-order logic over a countable tree with finitely many successor relations plus the relation of 'precedence' between nodes is decidable.

\section{$5 \quad$ Two perspectives: fragments or generalized semantics}

Now we need to compare $G A M$-semantics for $F O L$ with standard semantics for $G F$.

\subsection{Restricted syntax versus generalized semantics}

Giving each quantifier a guard is a syntactic restriction banning unbounded quantifiers. In this sense, $G F$ is a fragment of $F O L$. But there is also another perspective, where this move rather represents a semantic generalization. We now assume that quantification will normally take place in 'structured domains', where access from one group of objects to another must go via some connecting relation $R$ of some appropriate arity. Binary modal accessibility is a typical example. Standard models are the special case with $R$ the universal relation. Informally, then, there seems to be an analogy between

(a) using guarded formulas over standard models, and

(b) using arbitrary first-order formulas over suitably generalized models.

We will now show how these two approaches are equivalent in our setting.

\subsection{Reducing GAM logic to GF}

The following result is proved in Andréka, van Benthem \& Németi 1998, Section 5.

Definition Guarded translation.

Consider a $k$-variable first-order language $L\left\{x_{l}, \ldots, x_{k}\right\}$, with $R$ a new $k$-ary predicate. The translation guard takes $k$-variable first-order formulas $\phi$ to guarded first-order formulas $\operatorname{guard}(\phi)$ by relativizing all quantifiers to one and the same atom $R x_{1} \ldots x_{k}$. This translation works for polyadic first-order quantifiers just as well as single ones - and it even extends to the above substitution operators, if desired. There is also a matching semantic operation of model expansion. Let $(\boldsymbol{M}, V)$ be any general assignment model for $L\left\{x_{1}, \ldots, x_{k}\right\}$ - without the new predicate $R$. The standard model $G U A R D(M, V)$ is $\boldsymbol{M}$ viewed as a standard model, and expanded with the following interpretation: 
$R\left(d_{1}, \ldots, d_{k}\right)$ iff the assignment $x_{i}:=d_{i}(1 \leq i \leq k)$ is in $V$.

The following is easy to prove by induction on first-order formulas:

Lemma For all available assignments $s$ in $V$, and all $k$-variable formulas $\phi$,

$\boldsymbol{M}, \boldsymbol{V}, s \quad \mathrm{I}=\phi \quad$ iff $\operatorname{GUARD}(\boldsymbol{M}, \boldsymbol{V}), s \mathrm{l}=\operatorname{guard}(\phi)$

Here is a reduction of $G A M$-semantics to the Guarded Fragment.

Theorem For all first-order $k$-variable formulas $\phi$, the following are equivalent:

(a) $\phi$ is satisfiable in general assignment models,

(b) $\quad R x_{1} \ldots x_{k} \wedge \operatorname{guard}(\phi)$ is satisfiable in standard models.

Proof From (a) to (b), the Lemma supplies the reason. For the converse, suppose that $R x_{1} \ldots x_{k} \wedge \operatorname{guard}(\phi)$ has a standard model $\boldsymbol{M}$ under some variable assignment $s$. Now define a general assignment model $(N, V)$ by retaining only those variable assignments on $\boldsymbol{M}$ whose values for $x_{1}, \ldots, x_{k}$ stand in the relation $R_{\boldsymbol{M}}$. These include the assignment $s$ itself. Then it is easy to see that $N, V, s \mid=\phi$ as with the Lemma.

The translation also works directly for the full first-order language without the $k$ restriction, by a slightly modified translation. The converse direction was left open in Andréka, van Benthem \& Németi 1998. Marx 2001, van Benthem 2005 have solutions.

This translation is at the same time a faithful embedding of the earlier complete logic of general assignment models (Section 3) into the logic of the Guarded Fragment, which provides another explanation of its decidability.

\subsection{Reducing GF to GAM logic}

We need a translation again. But this time, it is not compositional in the earlier sense. The reason is the earlier failure of Existential Generalization (i) and Full Locality (ii) in general assignment models. We need these principles for some finite set of relevant formulas in the proof to follow, and hence we put them into the translation.

Definition GAM translation.

Let $\phi$ be any guarded first-order formula with a total set of variables $\boldsymbol{x}=x_{1}, \ldots, x_{k}$. Let $\operatorname{set}-u p(\phi)$ be the finite conjunction of all formulas of the following form

(i)' $\forall \boldsymbol{x}([\boldsymbol{u} / \boldsymbol{y}] \psi \rightarrow \exists y \psi) \quad$ where $\boldsymbol{u}, \boldsymbol{y} \subseteq \boldsymbol{x}$ and $\psi(z)$ is a subformula of $\boldsymbol{\phi}$ 


$$
\begin{array}{r}
\forall \boldsymbol{x}(\psi(z) \rightarrow \forall \boldsymbol{y} \psi(z)) \text { where } z, \boldsymbol{y} \subseteq \boldsymbol{x} \text { with } z \text { disjoint from } \boldsymbol{y} \\
\text { and } \psi(z) \text { is a subformula of } \phi
\end{array}
$$

The, not necessarily guarded, formula $\operatorname{gam}(\phi)$ is the conjunction $\phi \wedge \operatorname{set}-u p(\phi)$.

In particular, the prefixed polyadic universal quantifier $\forall x$ running over all relevant variables makes sure that the implications (i)', (ii)' hold throughout any general assignment model which has $\operatorname{set}-u p(\phi)$ true at any assignment at all.

Theorem For all guarded formulas $\phi$, the following are equivalent:

(a) $\phi$ is satisfiable in standard models,

(b) $\operatorname{gam}(\phi)$ is satisfiable in general assignment models.

Proof From (a) to (b), it suffices to note that any standard model for $\phi$ also satisfies $\operatorname{gam}(\phi)$, since the formulas in the second conjunct are universally valid. And standard models are general assignment models with a full set of assignments.

Next, from (b) to (a), let $\boldsymbol{M}, V, s \mathrm{l}=\operatorname{gam}(\phi)$. As in Section 3, this situation induces a quasi-model for $\phi$. Recall that the relevant formulas are all sub-formulas of $\phi$ plus their alphabetic variants with variables from $\boldsymbol{x}$. The types of the quasi-model are now all sets of relevant formulas true at the assignments in $V$. We must check the four clauses of the Definition. The first two follow directly by the truth definition for Boolean operations. The existential generalization clause holds for all types by the truth of conjunct (i)' in $\operatorname{set}-u p(\phi)$. And finally, the 'witness clause' (d) for existential quantifiers in suitably related types holds because of the truth condition for the existential quantifier in general assignment models plus the true transfer condition (ii)' in $\operatorname{set}-u p(\phi)$. Thus, the given guarded formula $\phi$ has a quasi-model - and hence it also has a standard model.

The same reasoning extends to the loosely guarded fragment $L G F$. Andréka \& Németi 2005 have some interesting variations and extensions. Marx 2001 proves several further results, including a characterization of the 'packed fragment' (a slight extension of $L G F)$. This is the largest fragment of first-order logic that is insensitive between evaluation in standard models and models relativized to some 'tolerance relation'. 


\section{Discussion}

The two main perspectives in this chapter are generalized models and static guards. Given the connections in Section 5, we merely discuss a few points about the latter.

The scope of guarding Guarding still has not become a general method. For instance, in modal logic, a lot of generality may be missed, since many results might work much more generally when basic modal logic is replaced by $G F$, the $\mu$-calculus by $L F P(G F)$, etc. Can the method of guarded fragments also be stated as a more general operation on logics? Van Benthem 2005 has an analysis of related methods for lowering complexity in second-order logic, going back to the fragment of 'persistent formulas' dating back to Orey in the 1950s (cf. Gallin 1975). These are invariant between Henkin general models and their associated standard models. Another challenge is guarding generalized quantifiers. We do not even know if the basic modal logic with a quantifier "for most successors of the current world" is decidable, despite partial results in Van der Hoek \& de Rijke 1993 and Pacuit \& Salame 2004. Perhaps most intriguingly, guards may help lower complexity in fixed-point logics, as we have seen in a number of cases.

Guarding lower down? Guards make sense, not just higher up from first-order logic, but also lower down in restricted formalisms. Kerdiles 2001 considers a language $C G$ of conceptual graphs which has only atoms, conjunction, and existential quantifiers. The complexity of the general consequence problem between such formulas is $N P$, but consequence between guarded $C G$-formulas is in $P$. This suggests that guarding can take the ' $N$ ' out of ' $N P^{\prime}$ ' sometimes, but the precise extent of this is unknown.

Algebra and logic once more We have seen in this chapter how ideas from algebra and algebraic logic can influence modal logic and first-order logic. Is there also a converse stream? It would be nice to see which of the various model-theoretic topics in this chapter make sense at an algebraic level. ${ }^{1}$ One major reason for going in this direction is this. With many concrete systems of modal logic being developed today (cf. the book van Benthem 2008), one feels that insights found there really live at some higher 'generic' abstraction level that can often be brought out better in an algebraic approach.

\footnotetext{
${ }^{1}$ For instance, it would be useful to have a better algebraic take on the fixed-point logics that crop up everywhere in contemporary pure and applied modal logic.
} 


\section{References}

H. Andréka, J. van Benthem \& I. Németi, 1998, 'Modal Languages and Bounded Fragments of Predicate Logic', Journal of Philosophical Logic 27, 217-274.

H. Andréka, I. Hodkinson \& I. Németi, 1999, 'Finite algebras of relations are representable on finite sets', Journal of Symbolic Logic 64, 243--267.

H. Andréka, J. Madarasz \& I. Németi, 2007, 'Logics of Relativistic SpaceTime', in M. Aiello, J. van Benthem \& I. Pratt, eds., Handbook of Spatial Reasoning, Springer, Dordrecht, 607-711.

H. Andréka \& S. Mikulas, 1993, 'The Completeness of the Lambek Calculus with Respect to Relational Semantics', Journal of Logic, Language and Information 3, 1-37.

H. Andréka \& I. Németi, 2005, private communication.

J. Barwise \& J. Seligman, 1995, Information Flow, Cambridge University Press, Cambridge.

J. van Benthem, 1983, Modal Logic and Classical Logic, Bibliopolis, Napoli.

J. van Benthem, 1988, ‘A Note on Jónsson's Theorem', Algebra Universalis 25, 391-393.

J. van Benthem, 1991, Language in Action, Categories, Lambdas and Dynamic Logic, North-Holland, Amsterdam and MIT Press, Cambridge (Mass.).

J. van Benthem, 1993, 'Modal Frame Classes Revisited', Fundamenta Informaticae 18: 2/3/4, 307-317.

J. van Benthem, 1995, 'What Makes Modal Logic Tick?”, manuscript, Institute for Logic, Language and Computation, University of Amsterdam. J. van Benthem, 1994, 'Dynamic Arrow Logic', in J. van Eyck \& A. Visser, eds., Logic and Information Flow, The MIT Press, Cambridge (Mass.), 15-29.

J. van Benthem, 1996A, 'Complexity of Contents versus Complexity of Wrappings', in M. Marx, M. Masuch \& L. Pólos, eds., Arrow Logic and Multimodal Logic, Studies in Logic, Language and Information, CSLI Publications, Stanford and Cambridge University Press, 203-219.

J. van Benthem, 1996B, Exploring Logical Dynamics, CSLI Publications, Stanford.

J. van Benthem, 1997A, 'Dynamic Bits and Pieces' and Pieces', Report LP-97-01, Institute for Logic, Language and Computation, University of Amsterdam. 
J. van Benthem, 1997B, 'Modal Foundations for Predicate Logic', Bulletin of the IGPL 5:2, 259-286, London and Saarbruecken (Proceedings WoLLIC 1995).

J. van Benthem, 1999, 'The Range of Modal Logic', Journal of Applied NonClassical Logics, vol. 9:2/3, 407-442.

J. van Benthem, 2001, 'Modal Logic in Two Gestalts', in M. de Rijke, H. Wansing \& M. Zakharyashev, eds., Advances in Modal logic, vol. II, Uppsala 1998, CSLI Publications, Stanford, 73-100.

J. van Benthem, 2005, 'Guards, Bounds, and Generalized Semantics', Journal of Logic, Language and Information 14, 263-279.

J. van Benthem, 2008, Logical Dynamics of Information Flow, book manuscript, Institute for Logic, Language and Computation, University of Amsterdam.

J. van Benthem \& D. Bonnay, 2008, 'Modal Logic and Invariance', Journal of Applied Non-Classical Logics 18:2-3, 153 - 173.

J. van Benthem \& K. Doets, 1983, 'Higher-Order Logic', in D. Gabbay and F. Guenthner, eds., Handbook of Philosophical Logic, vol. I., Reidel, Dordrecht, 275-329.

J. van Benthem \& M-C Martinez, 2008, 'The Stories of Logic and Information', in P. Adriaans \& J. van Benthem, eds., Handbook of the Philosophy of Information, Elsevier, Amsterdam.

J. van Benthem \& E. Pacuit, 2006, 'The Tree of Knowledge in Action', Proceedings Advances in Modal Logic, ANU Melbourne, 87-106.

M. van den Berg, 1996, The Internal Structure of Discourse, Dissertation, Institute for Logic, Language and Computation, University of Amsterdam.

P. Blackburn, M. de Rijke \& Y. Venema, 2000, Modal Logic, Cambridge University Press, Cambridge.

B. ten Cate, 2005, Model Theory for Extended Modal Languages, Dissertation, Institute for Logic, Language and Computation, University of Amsterdam. H-D Ebbinghaus \& J. Flum, 1995, Finite Model Theory, Springer, Berlin. S. Feferman \& G. Kreisel, 1969, 'Persistent and Invariant Formulas for Outer Extensions', Compositio Mathematica 20, 29-52.

D. Gallin, 1975, Systems of Intensional and Higher-Order Modal Logic, North-Holland, Amsterdam. 
R. Goldblatt \& S. Thomason, 1974, 'Axiomatic Classes in Propositional Modal Logic', in J. Crossley, ed., Algebra and Logic, Springer Lecture Notes in Mathematics 450, 163-173.

E. Grädel, 1999A, 'Decision Procedures for Guarded Logics', in Automated Deduction - Proceedings CADE 16, Lecture Notes in Computer Science 1632, Springer Verlag, Berlin, 31 - 51.

E. Grädel, 1999B, 'On the Restraining Power of Guards', Journal of Symbolic Logic 64, 1719-1742.

E. Grädel, 1999C, 'The Decidability of Guarded Fixed Point Logic', in JFAK. Essays Dedicated to Johan van Benthem on the Occasion of his 50th Birthday (J. Gerbrandy, M. Marx, M. de Rijke, and Y. Venema, eds.), http://turing.wins.uva.nl/ j50/cdrom/, Amsterdam University Press.

J. Groenendijk \& M. Stokhof, 1991, 'Dynamic Predicate Logic', Linguistics \& Philosophy 14, 39-100. Also in The Philosopher's Annual, 1992.

J. Halpern \& M. Vardi, 1989, ' The Complexity of Reasoning about Knowledge and Time, I: lower bounds'. Journal of Computer and System Sciences, 38(1), 195-237.

L. Henkin, 1949, 'Completeness in the Theory of Types', Journal of Symbolic Logic 15, 81-91.

J. Hintikka \& G. Sandu, 1997, 'Game-Theoretical Semantics', in J. van Benthem \& A. ter Meulen (eds.), Handbook of Logic and Language, Elsevier, Amsterdam, pp. 361-410.

W. Hodges, 2001, 'Formal Features of Compositionality', Journal of Logic, Language and Information 10, 7-28.

W. van der Hoek \& M. de Rijke, 1993, 'Generalized Quantifiers and Modal Logic', Journal of Logic, Language, and Information 2, 19-50.

E. Hoogland \& M. Marx, 2002, 'Interpolation and Definability in Guarded Fragments', Studia Logica 70, 373-409.

E. Hoogland, M. Marx \& M. Otto, 1999, 'Beth Definability for the Guarded Fragment', in H. Ganzinger, D. McAllester \& A. Voronkov, eds., Logic for Programming and Automated Reasoning, LPAR 6, Springer Lecture Notes in AI 1705, 273-285, Springer, Berlin. 
G. Kerdiles, 2001, Saying it with Pictures: a logical landscape of conceptual graphs, Dissertation DS-2001-09, Institute for Logic, Language and Computation, University of Amsterdam.

N. Kurtonina, 1995, Frames and Labels, A Modal Analysis of Categorial Inference, Dissertation, Institute for Logic, Language and Computation, University of Amsterdam.

E. Mares, 2003, Relevant Logic, a Philosophical Interpretation, Cambridge University Press, Cambridge.

M. Marx, 1995, Arrow Logic and Relativized Algebras of Relations, Dissertation, CCSOM and ILLC, University of Amsterdam.

M. Marx, 2001, 'Tolerance Logic', Journal of Logic, Language and Information 10, $353-373$.

M. Marx \& Y. Venema, 1997, Multi-Dimensional Modal Logic, Kluwer, Dordrecht.

S. Mikulas, 1995, Taming Logics, Dissertation, Institute for Logic, Language and Computation, University of Amsterdam.

R. Montague, 1970, 'Pragmatics and Intensional Logic', Synthese 22, 68-94.

I. Németi, 1985, 'Cylindric-Relativized Set Algebras Have Strong Amalgamation', Journal of Symbolic Logic 50:3, 689-700.

I. Németi, 1986, Free Algebras and Decidability in Algebraic Logic, Third Doctoral Dissertation, Mathematical Institute, Hungarian Academy of Sciences, Budapest.

I. Németi, 1995, 'Decidability of Weakened Versions of First-Order Logic', in Logic Colloquium 92, CSLI Publications, Stanford, 177-241.

I. Németi, 1996, 'Fine-Structure Analysis of First-Order Logic', in M. Marx, M. Masuch \& L. Pólos, eds., Arrow Logic and Multi-Modal Logic, CSLI Publications, Stanford, 221-247.

E. Pacuit \& S. Salame, 2004, 'Majority Logic', in D. Dubois, Ch. Welty $\&$ M-A Williams, eds., Proceedings of the Ninth International Conference (KR 2004), 598-605.

M. de Rijke, 1993, Extending Modal Logic, Dissertation, Institute for Logic, Language and Computation, University of Amsterdam.

J. Väänänen, 2007, Dependence Logic: A New Approach to Independence Friendly Logic, Cambridge University Press, Cambridge. 
F. Veltman, 1996, 'Defaults in Update Semantics', Journal of Philosophical Logic 25, 221-261. Also appeared in The Philosopher's Annual, 1997.

Y. Venema, 1991, Many-Dimensional Modal Logics, Dissertation, Institute for Logic, Language and Computation, University of Amsterdam.

Y. Venema, 1994, 'A Modal Logic for Quantification and Substitution', Logic Journal of the IGPL 2(1), 31-45.

Y. Venema, 1995, 'Cylindric Modal Logic', Journal of Symbolic Logic 60, 591-623.

Y. Venema, 1996, 'A Crash Course in Arrow Logic', in M. Marx, M. Masuch \& L. Pólos, eds., Arrow Logic and Multi-Modal Logic, CSLI Publications, Stanford, 3-34.

Y. Venema, 2006, 'Algebras and Coalgebras', in P. Blackburn, J. van Benthem \& F. Wolter, eds., Handbook of Modal Logic, Elsevier, Amsterdam, 331-426.

W. Wadge, 1975, 'A Complete Natural Deduction System for the Relation Calculus', Technical Report 5, University of Warwick. 